\title{
Caseous calcification of posterior mitral annulus: a forgotten benign condition mimicking atrial mass
}

\author{
Ahmad Abuarqoub, Vinod Kumar, Ali Atoot, Chirag Rana, Fayez Shamoon \\ Department of Cardiology, Saint Joseph's Regional Medical Center, Paterson, New Jersey, USA \\ Correspondence to: Ahmad Abuarqoub. 90 Arcadia Road Apt B, Hackensack 07601, New Jersey, USA. Email: ahmada6@hotmail.com.
}

\begin{abstract}
Caseous calcification of posterior mitral annulus is considered a variant of mitral annular calcification and accounts for less than $1 \%$ cases of mitral valve calcification (MAC). Usually benign and asymptomatic and has typical features on imaging studies but may contribute to unnecessary investigations and interventions in some patients, transthoracic echocardiography (TTE) is most useful tool in the diagnosis of this condition, whether it is associated with increased risk of atherosclerosis is unknown. We are presenting a case of caseous calcification of posterior mitral annulus that is discovered during elective coronary angiography in a patient with extensive history of coronary heart disease who had abnormal stress test.
\end{abstract}

Keywords: Caseous calcification of mitral (CCMA); mitral valve calcification (MAC); transthoracic echocardiography (TTE); trans-esophageal echocardiography (TEE)

Submitted Apr 18, 2018. Accepted for publication May 08, 2018.

doi: $10.21037 /$ atm.2019.10.114

View this article at: http://dx.doi.org/10.21037/atm.2019.10.114

\section{Introduction}

Caseous calcification of posterior mitral annulus is benign disorder and has an excellent outcome, could be misdiagnosed as cardiac tumors/vegetation, the typical imaging features should be recognized to avoid unnecessary surgical interventions.

\section{Case presentation}

A 77-year-old former smoker male with past medical history of diabetes, hypertension, COPD, coronary heart disease with history of CABG in 2013, and PCI of ramus intermedius in 2014. The patient was admitted with history of chest pain, that was sudden onset, retrosternal and heaviness in nature radiated to left shoulder that happened at rest and lasted for 20 minutes. The patient denied any other associated symptoms, but he claims having exertional chest pain in the past few weeks. EKG done and showed sinus rhythm, Q waves on inferior leads, poor R wave progression, which was the same as baseline. Nuclear stress test was done which showed medium sized reversible apical to mid anterior perfusion defect. Coronary angiogram done which showed severe native disease with Patent Grafts, also the patient was noted to have a mass like calcification at the mitral valve area (Figure 1). Echocardiogram was done which showed $2.5 \times 2.5 \mathrm{~cm}$ round echo dense mass with smooth borders and central areas of echo-lucency involving the posterior mitral annulus that is diagnostic for caseous calcification of posterior mitral annulus (Figures 2,3). The patient had an echocardiogram 2 years ago which was similar. The patient was sent home on medical therapy with planned follow up in outpatient cardiology clinic.

\section{Discussion}

Caseous calcification of mitral (CCMA) valve annulus is rare variant of mitral annular calcification (MAC). CCMA is described as a degenerative calcified necrotic changes of cardiac valve skeleton, which mainly occurs in location between the crest of posterior left ventricle muscle and posterior mitral annulus.

Caseous calcification has been reported in $0.5 \%$ to $1 \%$ 

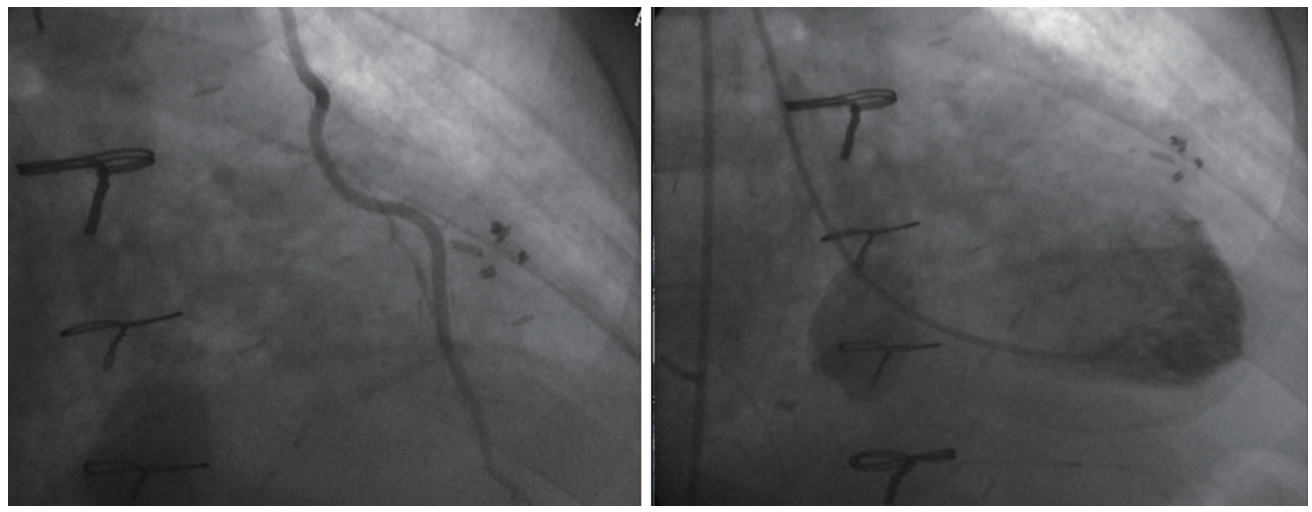

Figure 1 Showing patent LIMA to LAD, left ventriculogram showing normal EF with calcific mass seen at the area of the mitral valve. LIMA, left internal mammary artery; LAD, left anterior descending artery; EF, ejection fraction.

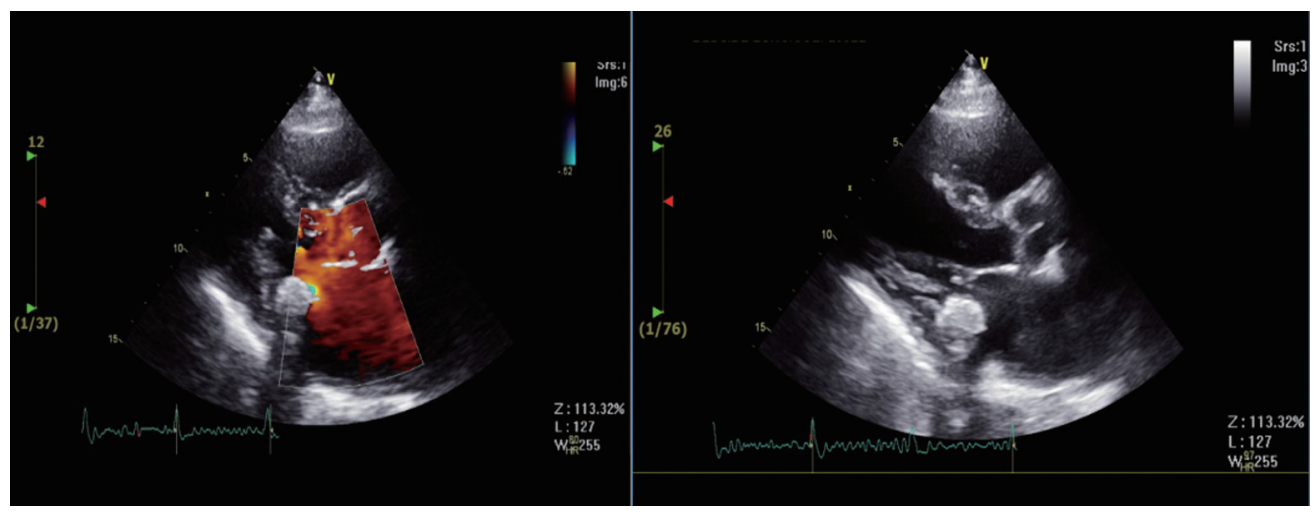

Figure 2 Parasternal long axis view with color Doppler showing round echo dense mass with smooth borders and central areas of echolucency involving the posterior mitral annulus.

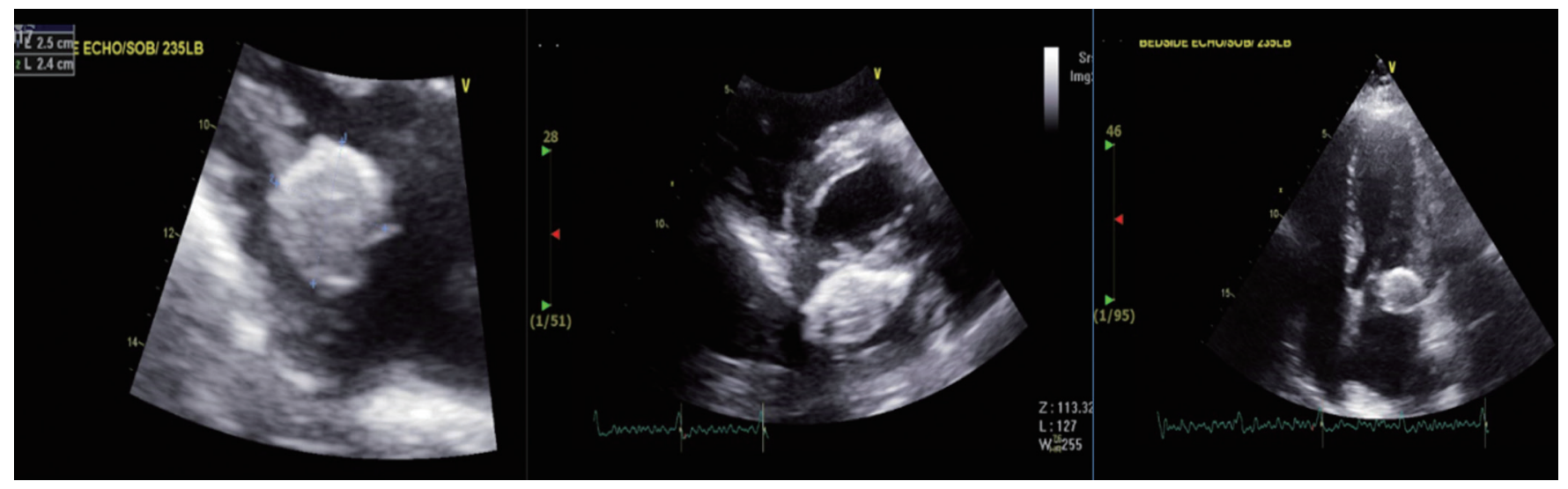

Figure 3 Echocardiogram showing $2.5 \times 2.5 \mathrm{~cm}$ echo dense mass, same is seen in short axis and apical four chamber views. 
of all mitral annular calcification (MAC), MAC is found in $10 \%$ population above the age of 50 (1).

CCMA was first described by Pmoerance in 1970 in one case series of autopsies, he described caseous calcified necrosis of mitral annulus in 7 out of 258 cases.

Caseous term is taken from caseous necrosis, which has been reported mainly in tuberculosis, in the setting of its cheesy appearance and so called caseous calcification. CCMA has typical echocardiographic appearance described as round, large semilunar, echo-dense mass with smooth borders with caseous putty like material surrounded by calcified envelope, can be seen as peri annular mass on trans-esophageal echocardiography (TEE).

CCMA differs from MAC in location, most cases of CCMA has been seen in posterior annular region of mitral valve unlike MAC were it mostly involves the mid-bases of posterior leaflets of mitral valve (2). Microscopic examination shows amorphous, acellular, basophilic material with chronic inflammatory reaction with macrophages, pathology is rarely required for diagnosis of CCMA since echocardiography is the gold standard.

Differential diagnosis of CCMA includes cardiac tumors, vegetation, thrombus or myxoma. Cardiac tumors may appear similar to caseous calcification on echocardiography. Caseous calcifications of mitral valve has been reported as benign and possess excellent prognosis (3). Most cases of CCMA reported with disorders of calcium and Phosphate metabolism such as in patients with chronic renal failure especially those on hemodialysis, and patients with hypercalcemia. Other associated conditions are hypercholesterolemia, hypertension, coronary artery disease and aortic atheromatous disease (4). Most patients with CCMA are asymptomatic, but very rarely patients may present with signs and symptoms of mitral insufficiency. Others could present with left ventricular outflow obstruction or mitral valve obstruction and rarely systemic embolization. In symptomatic cases surgical excision and valve repair is the treatment of choice (4).

In one study Dietl et al. reported risk of embolization and cardiovascular events in CCMA patients. They reviewed 1,052 articles and evaluated risk of embolization in 130 patients with CCMA and was $19.2 \%$ i.e., 25 of 130 cases had systemic embolization, which had higher risk for embolization as compared with MAC (11.8\% in MAC) (5).

\section{Acknowledgments}

None.

\section{Footnotes}

Conflicts of Interest: The authors report no conflicts of interest in this work.

Ethical Statement: The authors are accountable for all aspects of the work in ensuring that questions related to the accuracy or integrity of any part of the work are appropriately investigated and resolved. Written informed consent was obtained from the patient for publication of this case report and any accompanying images. A copy of the written consent is available for review by the Editor-inChief of this journal.

\section{References}

1. Chahal M, Temesy-Armos P, Stewart W. Big MAC: Caseous Calcification of the Mitral Annulus Referred for Possible Cardiac Tumor. Echocardiography 2011;28:E76-8.

2. Elgendy IY, Conti CR. Caseous calcification of the mitral annulus: a review. Clin Cardiol 2013;36:E27-31.

3. Zeina AR, Makhoul N, Nachtigal A. Mitral annulus caseous calcification imaged with 64-slice MDCT. Acute Cardiac Care 2009;11:121-2.

4. Kuwauchi S, Watanabe S, Abe K, et al. Caseous calcification of mitral annulus. Cardiovasc Diagn Ther 2013;3:108-10.

5. Dietl CA, Hawthorn CM, Raizada V. Risk of Cerebral Embolization with Caseous Calcification of the Mitral Annulus: Review Article. Open Cardiovasc Med J 2016;10:221-32.
Cite this article as: Abuarqoub A, Kumar V, Atoot A, Rana C, Shamoon F. Caseous calcification of posterior mitral annulus: a forgotten benign condition mimicking atrial mass. Ann Transl Med 2019;7(22):697. doi: 10.21037/atm.2019.10.114 